\title{
Commentary
}

\section{Hysterectomy - need of a paradigm shift}

\author{
Sunil Fernando ${ }^{1}$ \\ Sri Lanka Journal of Obstetrics and Gynaecology 2012; 34: 33-36
}

Hysterectomy is a common gynaecological surgical procedure performed for both benign and malignant conditions of the uterus and its appendages. Its main advantage is the elimination of the likelihood of a repeat gynaecological surgery especially if salpingoophorectomy was accompanied with it.

\section{Historical perspective}

The first elective vaginal hysterectomy was performed in 1883 by C. Langerbeck. It took another 50 years for the first abdominal subtotal hysterectomy which was done by Charles Clay of Manchester. Ernst Wertheim performed the first radical hysterectomy for cervical cancer in 1898, the same year in which Marie Curie discovered Radium, the other modality of treatment for the same condition. In 1902 Schauta introduced the first vaginal radical hysterectomy. Until 1929 the subtotal hysterectomy was the standard procedure and in that year Richardson is credited with performing the first total abdominal hysterectomy. Yet another 60 years elapsed when Harry Reich developed laparoscopic assisted vaginal hysterectomy and subsequently refined it to total laparoscopic hysterectomy.

With the advent of new techniques, and refining minimal access surgical equipment and energy sources the technique of hysterectomy has evolved over the last two decades. Although some of these procedures are viewed as technical adventures, the risk benefit ratio varies between different procedures. Albeit the new developments, the basic routes of hysterectomy remain the same, i.e. the abdominal (AH) and vaginal $(\mathrm{VH})$. The abdominal route is now being dominated by the newer laparoscopic techniques, ranging from laparoscopic assisted vaginal hysterectomy(LAVH), total laparoscopic hysterectomy (TLH) to single port laparoscopic hysterectomy(SIL) and robotic techniques. Although the preference for the route remains the prerogative of the attending clinician depending on clinical and technical factors,

\footnotetext{
${ }^{1}$ Department of Obstetrics and Gynaecology, North Colombo Teaching Hospital, Ragama.

Correspondence: Sunil Fernando

E-mail:sunilfer@sltnet.lk
}

there is overwhelming evidence that vaginal route is preferable to the abdominal route for benign conditions requiring hysterectomy whether or not oophorectomy is envisaged.

In most countries the abdominal route is the approach selected in majority of cases due to the simple reason that vaginal hysterectomy for a non prolapsed uterus is more technically demanding, and especially so with the increase in size of the uterus beyond 10-12 weeks of gravid uterus, and the presence of adnexal pathology. Ovaries being less accessible through vaginal route due to their higher position and less mobility of the infundibulo pelvic ligaments, whenever there is a need for oophorectomy, it is regarded as an excuse for performing abdominal hysterectomy.

In the United States about 600,000 hysterectomies are performed annually and more than two thirds are performed abdominally $(\mathrm{AH})$. Although audits on this are scarce, in Sri Lanka the abdominal route must be preferred in well over $90 \%$ for benign disease without a prolapse. Although the technique of non descent vaginal hysterectomy is more challenging and has a long learning curve, it remains to be seen what else is best for the patient. The patient demand for a particular procedure is based on the recent evidence for which most of our patients have an access to. Informed patient choice is one of the principles on which we have to adopt our surgical techniques in years to come. Hence it is imperative that we as gynaecologists try to achieve the technical expertise of vaginal procedures as well as other minimal access procedures because of their better safety profile compared to other techniques. In a position statement published in the Journal of Minimally Invasive Gynaecology ${ }^{1}$, The American Academy of Gynaecologic Laparoscopists (AAGL) recommends minimally invasive surgical approaches such as vaginal hysterectomy and laparoscopic hysterectomy for benign uterine disease.

\section{Advantages of minimal access hysterectomy}

In a multicenter study comparing LAVH and $\mathrm{TAH}$, the mean operating time was significantly longer for LAVH than VH (179.8 vs. 146.0 minutes) and significantly shorter hospital stay (2.1 days) and convalescence (28.0 days) for VH than LAVH (4.1 days and 38.0 days, respectively ${ }^{2}$. Whereas $\mathrm{AH}$ is 
associated with two incisions, one being abdominal and the other being vaginal, the vaginal route utilizes only vaginal incision thereby minimizing all the complications related to the abdominal incision, namely post-operative pain, wound infection, dehiscence and ventral hernias. Less post-operative pain allows mobilization of the patient early there by reduces the risk of respiratory infections and deep vain thrombosis.

The mean discharge time was longer for $\mathrm{LAVH}$ than for $\mathrm{VH}(72 \pm 4.2$ vs. $48 \pm 2.6 \mathrm{~h} ; \mathrm{P}=0.00)$. VH resulted in shorter times for paralytic ileus (19 \pm 3 vs. $26 \pm 3 \mathrm{~h}$; $\mathrm{P}=0.00)$ and surgery $(71 \pm 3$ vs. $129 \pm 7 \mathrm{~min} ; \mathrm{P}=0.00)$. The intraoperative blood loss was less with $\mathrm{VH}(186.0 \pm 52$ vs. $362.7 \pm 65 \mathrm{~mL} ; \mathrm{P}=0.00)^{3}$.

$\mathrm{VH}$ is feasible with regional anaesthesia and combining with an epidural offers best analgesia for the first post-operative day. This is feasible since regional anaesthesia is always used in non descent $\mathrm{VH}$. Since there is no bowel handling during surgery, the risk of post-operative paralytic ileus is minimal. The risk of return to the operative theatre is remote, hence patients start solid diet within 2-4 hours of the surgery.

Sometimes the pain following $\mathrm{VH}$ is due to the use of vaginal retractors which may cause overstretching of the tissues. Using narrow and long specula obviate the need for more pain relief post operatively. In a non-prolapse hysterectomy, approximating the two cardinal ligaments and tying them together could result in more post-operative pain. This is a step that could be safely avoided since the supporting structures are strong and there is no need for repair to prevent future vault prolapse. Blood loss at surgery also remains to be minimal due to less tissue disruption. Sedentary workers are able to return to work in 1-2 weeks. Since there is a high risk of wound infection in obese patients ${ }^{5} \mathrm{VH}$ offers the best solution for them. Because of lower morbidity and speedy recovery and short hospital stay, it is a cost -effective procedure as well.

\section{Large uterus for vaginal hysterectomy}

Leiomyomas can grow to large volumes and Nappi et $a l^{4}$ in Italy described a myoma of the uterus weighing $27.7 \mathrm{~kg}$. While these are obviously dealt with abdominal route, uteri up to 28 weeks size (1000 g) are amenable for vaginal surgery.

Volume-reducing techniques such as hemisection (bivalving), intramyometrial coring, (Lash procedure), wedge incision (V-type), morcellation and enucleation of myomas of a large uterus has circumvented the main disadvantage of vaginal route for hysterectomy, viz the inadequate space. In all these cases the possibility of malignancy should be excluded to prevent intraabdominal contamination. These steps could be performed once the main blood supply to the uterus is ligated and the colour of the uterus changes and assumes a pale hue and only after the peritoneal cavity is opened either anteriorly or posteriorly. In a uterus with multiple fibroids, removal of fibroids achieves the much needed space within a short time. It is important to pay attention to the anterior and posterior uterine walls to minimize damage to the rectum and the bladder. Care should be taken in removal of a large uterus with adenomyosis, since it is difficult to morcelate these hard uteri and extra force used to achieve this may damage the adjacent structures.

All these uteri require size reduction by one of the above methods. The factor that determine mostly for the time taken for the procedure is the ease with which this was achieved. Adenomyotic uterus is so hard that it takes a longer time for removal. Size reduction procedures should be commenced only after uterine arteries are tied to minimize bleeding. One should try to go tying pedicles as high as possible before dissecting the uterus. In pedicle suturing, holding the needle at the tip of the needle holder at a sharp angle will help to achieve higher placement of sutures. Up down or down up needle entry method could be used depending on the space available

Traditionally the surgery starts with opening into the pouch of Dougles through posterior colpotomy, but there are situations where this is not possible due to endometriotic adhesions or nodular endometriosis in the recto vaginal septum. In such situations surgery could be continued by dissecting the adhesions vaginally until one reaches the top of the adhesions and bare uterine wall, carefully following the correct tissue planes both by sharp and finger dissections. Dissecting close to the posterior uterine wall is a safe technique to avoid injuries to the rectum and the rectal adhesions could also be assessed with digital rectal examination.

\section{Training in $\mathrm{VH}$}

Having observed and assisted in these procedures one should start with slightly enlarged uteri with some degree of early prolapse in parous patients without previous abdominal surgeries including caesarean section where significant amount of bladder adhesions are observed frequently. When confidence is achieved, one should venture into more difficult situations and gain significant skills. Evaluation of accessibility and mobility of uterus is determined by the operator experience. 
Through persistent and inquisitive approach one will soon acquire a steep learning curve. Transferring skills is achieved through observing, assisting and being fostered. Maintaining an audit of the procedures and regular reflection of difficult situations is imperative and help in improving one's skills as well. There should be no hesitancy for conversion to an abdominal procedure and this should not take away one's confidence.

Developing a team of assistants including the nursing staff is important to perfect the procedure and to save time. Post-surgical care staff should be skilled to maintain epidural analgesia.

\section{Our experience with VH for very large uterus}

Out of 92 nonprolapse vaginal hysterectomies performed by the author from March 2010 to March 2012, 26 were with the size over 16-28 weeks gravid uterus. The indications for hysterectomy for these 26 cases included abnormal uterine bleeding in 12 patients, dysmenorrhoea and dyspareunia 6 patients, detection of an abdominal mass in 6 patients and urinary symptoms in 2 patients.

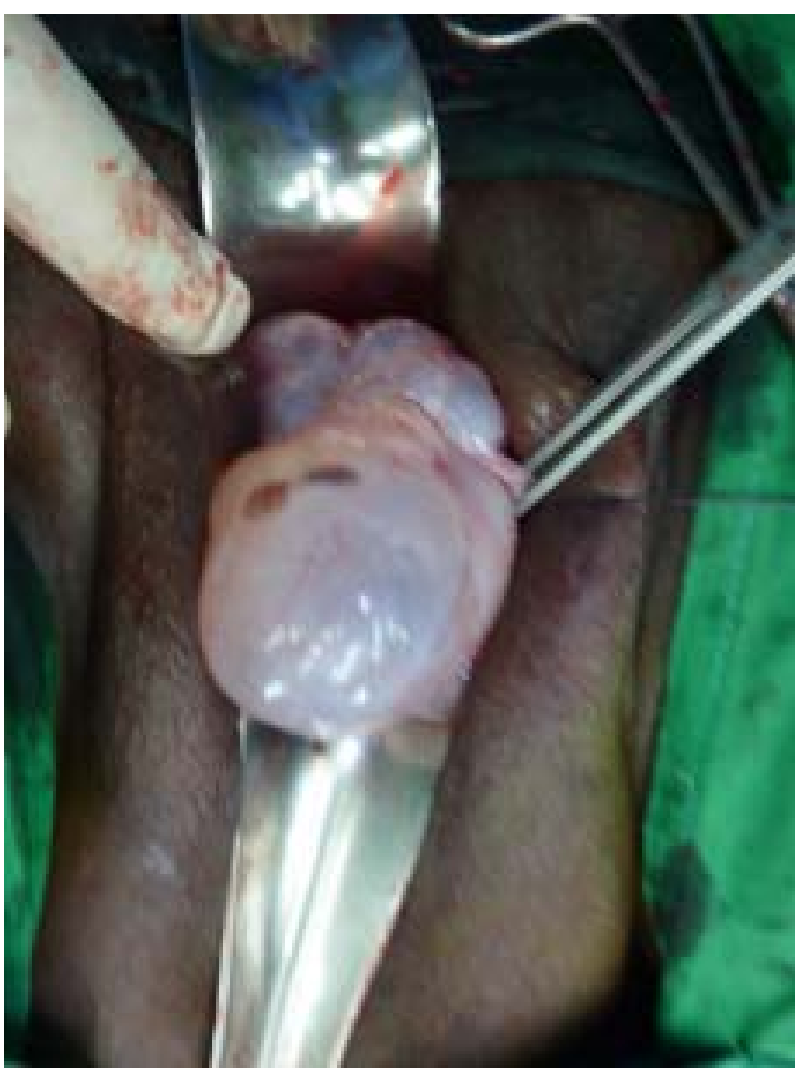

Figure 2. Vaginal oophorectomy for benign ovarian tumour.
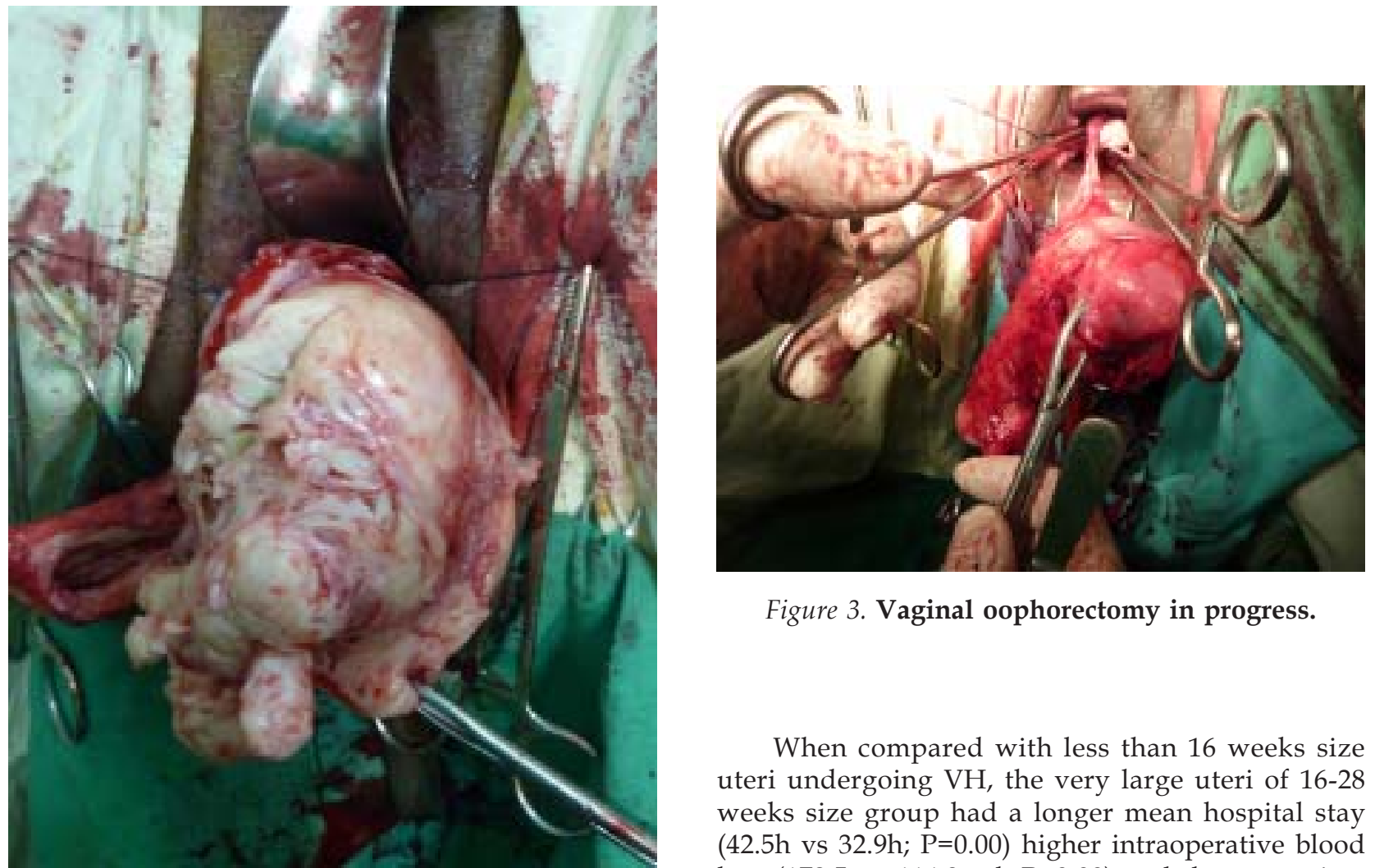

Figure 3. Vaginal oophorectomy in progress.

Figure 1. Vaginal hysterectomy for a very large uterus with multiple myomas. uteri undergoing $\mathrm{VH}$, the very large uteri of 16-28 weeks size group had a longer mean hospital stay (42.5h vs 32.9h; $\mathrm{P}=0.00$ ) higher intraoperative blood loss (178.5 vs $114.3 \mathrm{ml} ; \mathrm{P}=0.00)$ and the mean time taken for surgery was longer (57.5 vs 110.3 mins; $\mathrm{P}=0.00)$. 


\section{Complications: VH for very large uterus} (16-28 weeks)

\begin{tabular}{ll}
\hline Haematoma requiring drainage & 1 \\
Vault infection & 3 \\
Urinary tract infection & 2 \\
Post-operative blood transfusion & 0 \\
Damage to bladder and bowel & 0 \\
Paralytic ileus & 1 \\
Vaginal wall tears requiring suturing & 2 \\
\hline
\end{tabular}

Out of 92 patients three VHs were intraoperatively converted to abdominal after clamping the uterine arteries due to adhesions between the bowel and body and fundus of the uterus and one due to dense adhesions to the anterior abdominal wall following two caesarean sections.

Bilateral salpingoophorectomy, when it was intended, was successfully done in 95\% (56 patients) and two had to be abandoned due to extreme atrophy and adhesions to the lateral pelvic wall. One patient had to undergo laparoscopic omentectomy and ipsilateral oophorectomy following $\mathrm{VH}$ and unilateral salpingoophorectomy done for a tubo-ovarian mass with an enlarged uterus which revealed to contain a tubal malignancy on histology. One patient who underwent $\mathrm{VH}$ and BSO was diagnosed as having stage $1 \mathrm{~A}$ endometrial carcinoma where the preoperative histology revealed complex hyperplasia of endometrium.

\section{Conclusion}

Minimal access vaginal hysterectomy is the mainstay in this commonly performed gynaecological procedure, which could be performed with slight modification of the routinely available instruments in any gynaecology theatre. Vaginal removal of a larger uterus should be undertaken only with great care and only after mastering the technique adequately. Intraoperative conversion to abdominal procedure may become necessary if the uterine mobility or descent is not appreciated after uterosacral cardinal complex is dissected. Great care should be taken in selecting patients for $\mathrm{VH}$ especially to exclude malignancy of the genital tract.

\section{References}

1. Journal of Minimally Invasive Gynaecology, online publication, November 16, 2010.

2. Summitt RL Jr, Stovall TG, Steege JF, Lipscomb GH. A multicenter randomized comparison of laparoscopically assisted vaginal hysterectomy and abdominal hysterectomy in abdominal hysterectomy candidates. Obstet Gynecol 1998; 92(3): 321-6.

3. Sesti F, Ruggeri V, Pietropolli A, Piccione E. Laparoscopically assisted vaginal hysterectomy versus vaginal hysterectomy for enlarged uterus. Journal of the Society of Laparoendoscopic Surgeons 2008; 12(3): 246-51.

4. Nappi L, Matteo M, Giardina S, Rosenberg P, Indraccolo U, Greco P. Management of uterine giant myoma. Gynecol Obstet 2008; 278: 61-63.

5. Soper DE, Bump RC, Hurt WG. Wound infection after abdominal hysterectomy: effect of the depth of subcutaneous tissue. Am J Obstet Gynecol 1995; 173(2): 465-9, 469-71. 